\title{
Shielding analysis in the design phase of the new Emergency Operation Facility for Tihange Nuclear Power Plant
}

\author{
Gilles Genard ${ }^{1, a}$, Romain Portal ${ }^{1}$, Virginie Bouchat ${ }^{1}$ and Serge Vanderperre ${ }^{1}$ \\ ${ }^{1}$ Radwaste management - Decommissioning - Radiation protection, Tractebel, Ariane avenue 7, B1200 Brussels - Belgium
}

\begin{abstract}
In the framework of the design studies for a new Emergency Operation Facility (EOF) for Tihange NPP, radiation protection analyses are needed to comply with effective dose rate criteria. In this aim, the shielding performed by, at the one hand, external walls and roof of the building and, on the other hand, internal walls, has been sized by means of MicroShield calculations. This paper explains how the calculations for external walls, doors, roof, floor and internal walls are made. The assumptions on the source terms and on the source geometry as well as the way the shielding is determined and the results of the sizing are presented.
\end{abstract}

\section{Introduction}

In the design phase of any new building on a nuclear site, such as a Nuclear Power Plant (NPP), considering the radiation protection aspects is mandatory to ensure workers and general population protection against ionizing radiations hazards. This is even more the case if radioactive contamination in the surrounding atmosphere of the building is anticipated or if radioactive sources are located in the building itself. As a consequence, designing shielding is necessary. At first, this shielding can be achieved by the building walls, the external and internal ones.

In the framework of the basic design studies for a new Emergency Operation Facility (EOF) for Tihange NPP, such studies are necessary. Indeed, this facility would be built on the site of the NPP and, due to its objective, which is to be used after a nuclear accident on one of the three units of the nuclear site, radioactive sources are expected around and inside the building. The basic design foresees that the inside of the EOF is subdivided in two zones: a Non Habitable Zone (NHZ) and a Habitable Zone (HZ). Consequently, appropriate shielding has to be put in place to ensure the safety of the workers managing the severe accident from the HZ of the EOF.

The source to consider for the shielding performed by external walls is the atmospheric contamination released around the building from the venting of one of the containment units through the filtered vent of this unit and the natural leak through the containment. The main source expected inside the building, to be considered to size internal walls, is due to the Heating, Ventilation and Air Conditioning (HVAC) filters that process external atmospheric contamination (and are therefore contaminated by the aforementioned atmospheric release) before supplying the air in the habitable zone of the building.

In the following, the paper tackles the study for the determination of minimum thicknesses of external walls, roof and door and of the internal walls, door and floor of the building.

\section{Initial source term}

The definition of the initial source term has been conducted by considering only one nuclear power unit affected by a severe accident. Note that, even if several units were affected by a severe accident, the wind cannot direct all the radioactive releases towards the EOF. To be conservative and knowing that the NPP units are similar enough (i.a. in terms of produced power) to present relatively comparable releases, the unit closest to the new EOF location has been taken into consideration.

Furthermore, in a conservative way, the wind is considered to be blowing the radioactive releases directly on the EOF. The wind speed is taken at $1.7 \mathrm{~m} / \mathrm{s}$, which is a relatively weak speed, limiting the atmospheric dispersion. No other aspect of the weather conditions (rain or dry atmosphere) is taken into account.

To determine the initial source term, several scenarios have been considered:

- The release of activity from the filtered vent,

- The release of activity through natural leaks of the containment unit,

- The release of activity from the filtered vent and through natural leaks of the containment unit.

For each scenario, activities of each radionuclide released in the atmosphere every 3 minutes during 10 days were calculated by means of the MELCOR code [1]. The period of 3 minutes is the time needed for the

\footnotetext{
a Corresponding author: gilles.genard@tractebel.engie.com
} 
contamination released from the vent chimney to reach the EOF with the considered wind speed.

The initial source term for each scenario, defined by the MELCOR code, features 1038 radionuclides. For each of these radionuclides, the released activities in function of time after initiation of the first venting, integrated during 3 minutes, are determined.

\section{External shielding sizing}

In the basic design, the EOF is $46 \mathrm{~m}$ long, $20 \mathrm{~m}$ wide and $4 \mathrm{~m}$ high and has a cuboid shape. Its external shielding will be assured by the four external walls and the roof, both in concrete, and, potentially, one external door in steel in one of those walls. The wall thickness will be identical for all four sides of the new building.

The dose rate calculations are performed by means of the MicroShield v5.05 code [2]. The latter is a calculation code, based on the "single point kernel" technique, which can determine gamma dose rate at a calculation point according to simple source and shielding geometries.

\subsection{Sizing criterion}

The shielding is meant to keep the effective dose rate below $1 \mathrm{mSv} / \mathrm{h}$ at $50 \mathrm{~cm}$ from the walls, which is considered to be the average walking distance from a wall, and at $1.30 \mathrm{~m}$ high, which is the average height of the personal dosimeters of the workers.

\subsection{External walls and roof}

\subsubsection{Methodology}

In order to ensure that the radiological criterion is complied with, three relevant locations in the EOF are considered (Points 1, 2 and 3, see Figure 1). The effective dose rate calculations are performed for each of these locations.

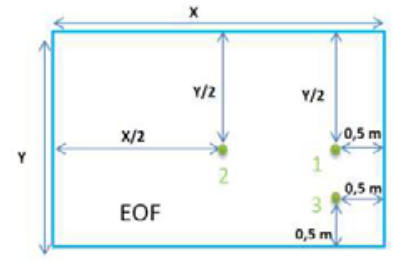

Top view

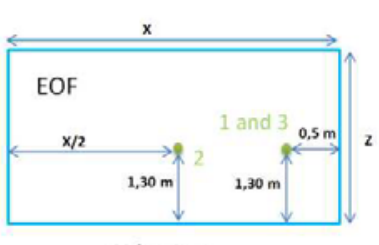

Side view
Figure 1. Schematic drawing of the calculation points in the EOF for external shielding sizing.

Point 1 is used to define the external wall thickness. To consider a conservative approach and keeping in mind that all walls will have the same thickness, this Point 1 is placed at $0.5 \mathrm{~m}$ of the nearest wall facing the potentially affected reactor building (RB) and in the middle of the same wall (to maximise dose rate). Point 2 is placed at the centre of the building and is used for the calculation of the roof thickness. Point 3 is placed in a corner at $0.5 \mathrm{~m}$ of two walls. This third point was chosen to check that workers located in this particular configuration are not exposed to a dose rate higher than the sizing criterion.

In order to comply with the sizing criterion of $1 \mathrm{mSv} / \mathrm{h}$ in the EOF, it is considered, for an initial stage, that half of the dose rate contribution inside the new building arises from the radiation through the lateral external walls and the other half of dose rate contribution arises from the radiation through the roof. Therefore, the calculations start by independently determining the minimal thicknesses for the external walls and roof by assuming that dose rates at Point 1 and Point 2 are below half of the sizing criterion $(<0.5 \mathrm{mSv} / \mathrm{h})$. Afterwards, the total dose rate due to the contributions arising from all directions is calculated at Points 1 and 3 to verify the compliance with the $1 \mathrm{mSv} / \mathrm{h}$ criterion.

Therefore, the calculation steps are:

- Step 1: performing the sizing of the external wall (YZ plane in Figure 1) on the basis of the effective dose rate at Point 1 due to the contribution of the external radiation through this wall and by assuming that this dose rate contributes for half of the sizing criterion $(<0.5 \mathrm{mSv} / \mathrm{h})$. The thickness obtained for this wall (side closest to the potentially impacted NPP unit) is then applied to the external walls of the three other building sides, conservatively,

- Step 2: performing the sizing of the roof (XY plane in Figure 1) on the basis of the effective dose rate at Point 2 due to the contribution of external radiation through the roof and by assuming that this dose rate contributes for half of the sizing criterion $(<0.5 \mathrm{mSv} / \mathrm{h})$,

- Step 3: verification of the compliance with the sizing criterion $(<1 \mathrm{mSv} / \mathrm{h})$ for Point 1 by calculating the total effective dose rate due to external radiation through the roof and the walls, except the wall on the side opposite to the releases (since this wall is the furthest from the potentially impacted NPP unit and the internal walls will also act as a shielding for the irradiation coming from there),

- Step 4: verification of the compliance with the sizing criterion $(<1 \mathrm{mSv} / \mathrm{h})$ for Point 3 by calculating the total effective dose rate due to the external radiation through the same directions as Step 3.

To obtain the minimal thickness needed for the shielding, a curve representing the effective dose rate in function of the shielding thickness is obtained thanks to various calculations with different shielding thicknesses but the same source term. Then, the shielding thickness is determined as the first thickness value in centimetre beyond which the effective dose rate is below the considered sizing criterion $(0.5$ or $1 \mathrm{mSv} / \mathrm{h}$ depending on the step as explained previously).

\subsubsection{Materials}

The source term being an external one, it is composed of air with a density of 0.00122 . The walls and roof are considered to be in concrete with a density of 2.35 . The detailed compositions are the standard ones of MicroShield. 


\subsubsection{Source terms}

For sizing external walls and roof, the retained activity for the MicroShield calculations is the maximum activity over time released from the filtered vent and through natural leaks of the containment unit (third scenario defined in Section 2). However, the release through natural leakage was proven to be negligible compared to the release from the filtered vent.

Due to the distance between the vent of the RB and the EOF, a dispersion of the released activity occurs. As illustrated in Figure 2, it is assumed that the total contamination vented during the time to reach the EOF is uniformly spread into two volumes: a cuboid volume and a pyramidal volume.

The cuboid volume, surrounding the EOF, is delimited by a base area which has the EOF dimensions increased by $5 \mathrm{~m}$ on all sides (see upper part of Figure 2) and a height identical to the one of the venting chimney (see lower part of Figure 2). This height is conservative since the radioactive releases, hotter than atmospheric air, should be dispersed at higher height than the vent of the $\mathrm{RB}$ and this decreasing effect on the source term is neglected here. The pyramidal volume joins the upper part of the venting chimney to the lateral side of the previously described cuboid volume. This volume is also very conservative considering the very low distribution angle of the releases.

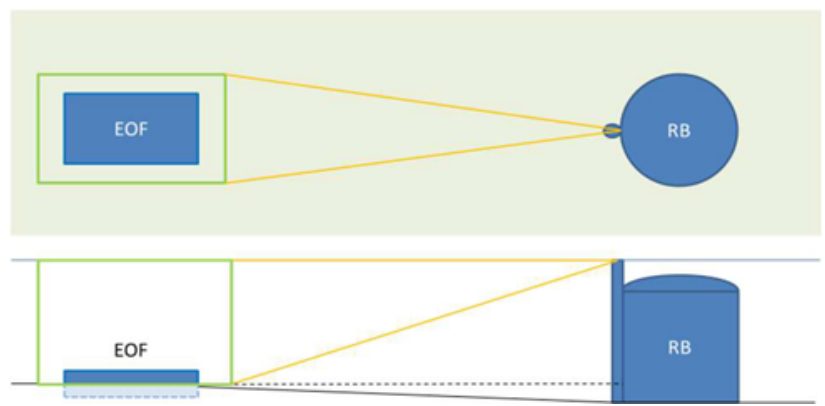

Figure 2. Schematic drawing of the source volume around the EOF coming from the vent of the RB.

For each of the 1038 radionuclides, the maximum activity selected among the activities calculated by the MELCOR code are then converted into activity concentration by taking into consideration the total volume obtained by adding the cuboid and pyramidal volumes.

Finally, the initial list of 1038 radionuclides is reduced down to 8 radionuclides on the basis of a general methodology developed by Tractebel [3] aiming at selecting the most relevant ones (in function of the magnitude of their activity, their fictive weight and their gamma ray energies). The selected radionuclides are radionuclides of krypton and xenon.

\subsubsection{Models}

The MicroShield code only enables using source volumes with simple geometries, which implies performing simplifications in a conservative way. To calculate the effective dose rates at the three calculation points, the cuboid and pyramidal source volumes are fractionated in five different volumes, as represented in Figure 3.
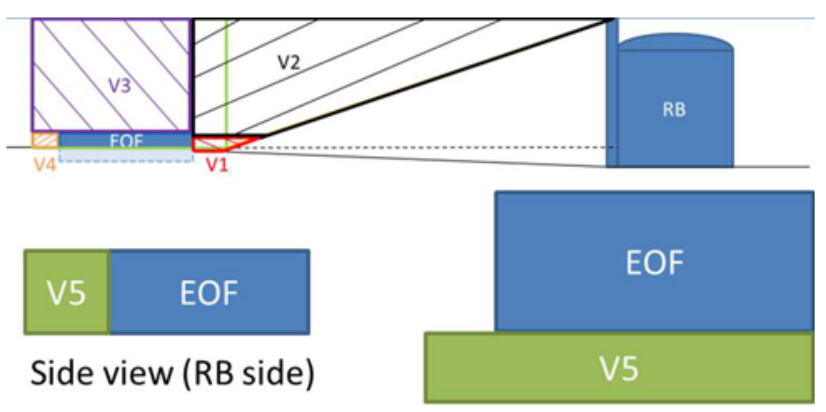

Top view

Figure 3. Definition of the volume fractions (V1, V2, V3, V4 and V5) of the source volumes.

Each model in the MicroShield code is defined by the shielding geometry and the volume fraction of the considered source term. However, MicroShield does not enable the modeling of a shielding whose height and width are smaller than those of the source volume. Furthermore, as it is not possible to model pyramidal source volume with this code, a cuboid source with an equivalent volume and having the same activity concentration must be used.

Consequently, the different models are:

Model 1, used for dose rate calculations at Point 1 and Point 3, shown in Figure 4:

- Source geometry is a cuboid with a width equal to the one of V1 and V2 (length of the EOF wall $(20 \mathrm{~m})$ with $5 \mathrm{~m}$ added on both sides), a height equal to the sum of the ones of $\mathrm{V} 1$ and $\mathrm{V} 2$ and a length determined to get a volume equivalent to $\mathrm{V} 1+\mathrm{V} 2$,

- Shielding is a concrete wall with a varying thickness,

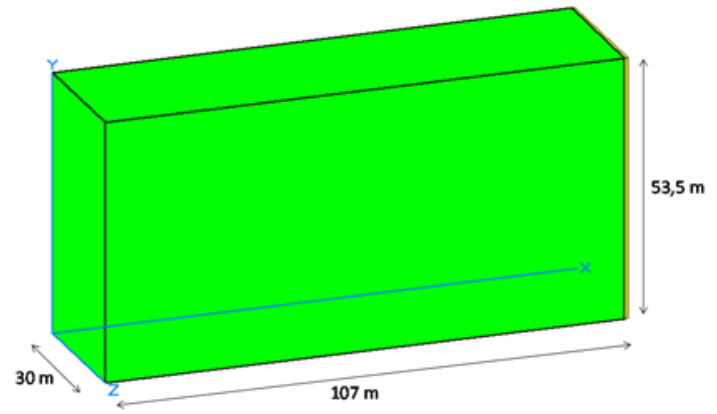

Figure 4. Representation of Model 1 as used in MicroShield.

Model 2, used for dose rate calculation at Point 2:

- Source geometry is a cuboid with a width equal to the one of V3 (length of the EOF roof $(20 \mathrm{~m})$ with 5 m added on both sides), a height equivalent to the one of V3 (the height of the vent chimney at which the EOF height was subtracted) and a length determined to get a volume equivalent to $\mathrm{V} 2+\mathrm{V} 3$,

- Shielding is a concrete roof with a varying thickness, Model 3, used for dose rate calculations at Point 1 and Point 2:

- Source geometry is V3,

- Shielding is a concrete roof with a thickness defined by Step 2 of the methodology, 
Model 4, used for dose rate calculations at Point 1 and Point 3:

- Source geometry is V5,

- Shielding is a concrete wall with a thickness defined by Step 1 of the methodology.

\subsubsection{Results}

Figure 5 presents the results of the calculation for Step 1 with dose rates calculated for several wall thicknesses. A curve is fitted on those calculated points and its intersection with the horizontal sizing criterion line $(0.5 \mathrm{mSv} / \mathrm{h})$ provides a minimal wall thickness of $49 \mathrm{~cm}$. A similar curve is established for Step 2 in order to define a minimal roof thickness.

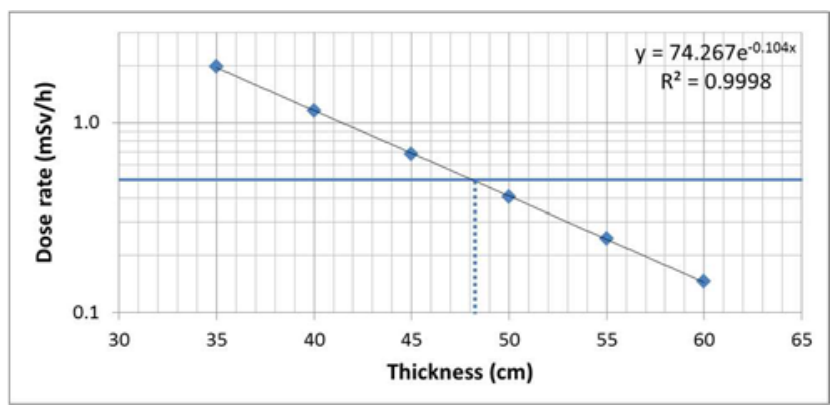

Figure 5. Dose rate evolution relative to the concrete thickness at Point 1 (the horizontal line being the $0.5 \mathrm{mSv} / \mathrm{h}$ sizing criterion).

The results of the application of the methodology mentioned in Section 3.2.1 can be summarized as follows:

- Step 1: minimal wall thickness is $49 \mathrm{~cm}$,

- Step 2: minimal roof thickness is $50 \mathrm{~cm}$,

- Step 3: effective dose rate at Point 1 is $0.74 \mathrm{mSv} / \mathrm{h}$, which is well below $1 \mathrm{mSv} / \mathrm{h}$,

- Step 4: effective dose rate at Point 3 is $0.68 \mathrm{mSv} / \mathrm{h}$, which is well below $1 \mathrm{mSv} / \mathrm{h}$.

Consequently, for this initial stage, the minimum thicknesses for the walls and roof are $49 \mathrm{~cm}$ and $50 \mathrm{~cm}$, respectively.

\subsection{External door}

\subsubsection{Methodology}

The source term, the conditions and the models to be considered are the same as those considered for the walls thickness calculations. As a consequence, the necessary equivalent thickness of an external stainless steel door can be estimated based on the shielding thicknesses obtained for the concrete walls, by using the respective linear attenuation coefficients of concrete and steel.

As the way to size the wall shielding is conservative, in particular if the external door is foreseen on a building side that is not facing the venting chimney (see $\mathrm{XZ}$ plane in Figure 1), an optimisation process can be applied in order to reach a more realistic thickness for the door. It is considered that the contribution coming from external radiation through the concerned wall side (including through the door) could be increased up to an effective dose rate at Point 1 equal to the $1 \mathrm{mSv} / \mathrm{h}$ criterion instead of $0.74 \mathrm{mSv} / \mathrm{h}$.

\subsubsection{Results}

The thickness of the wall where the external door will be integrated has been decreased from $49 \mathrm{~cm}$ to $18 \mathrm{~cm}$ of concrete. This induces a minimal stainless steel thickness estimated and rounded up to $5 \mathrm{~cm}$.

\section{Internal shielding sizing}

The previous calculations have enabled to determine a thickness for the external walls, roof and external door of the EOF. However, they did not take into account the potential presence of radioactive sources inside the building.

The most important potential radioactive sources inside the EOF are the filters of the HVAC system. Two types of filters are featured in this system: charcoal filters for iodine elements and absolute filters for particulates. Both filter types are installed in two successive boxes (see Figure 6, the FA box to filter the Fresh Air from outdoors and the HZ box to filter the part of the filtrated fresh air used for the $\mathrm{HZ}$ ventilation). As represented in Figure 6, into each of these boxes, the incoming air passes successively through the charcoal filters and the absolute filters. The accumulation of activity on those filters only occurs when radioactive contamination is present outside the EOF and causes the apparition of an internal radiation source which has to be considered for internal shielding sizing.

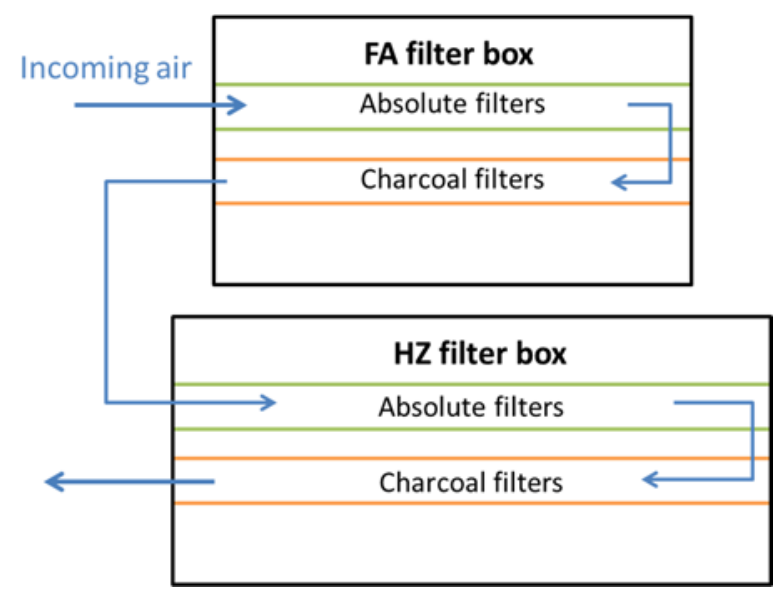

Figure 6. Schematic drawing of the FA and HZ filter boxes (side view).

The dose rate calculations for the internal shielding sizing are also performed by means of the MicroShield code, in its version 8.03 in this case [4].

\subsection{Sizing criterion}

$1 \mathrm{mSv} / \mathrm{h}$ is still the maximum dose rate at which workers can be exposed in the HZ. However, a (maximum) contribution of $0.74 \mathrm{mSv} / \mathrm{h}$ is already to be taken into 
account due to the contaminated air surrounding the EOF as detailed previously. Therefore, the criterion to use for the internal shielding sizing is $0.26 \mathrm{mSv} / \mathrm{h}$ at $0.5 \mathrm{~m}$ of the walls.

\subsection{Methodology}

The filtration boxes of the HVAC system are located in a room inside the NHZ of the EOF. As the main internal source terms arise from those filtration systems, the shielding has to be calculated for the wall separating this room from the habitable part of the new building. Therefore, the sizing calculation is related to the thickness of this wall and not to all other internal walls that do not have a shielding function. The floor of this room is also considered as a shielding, as a part of the HZ is located on the lower level, and its thickness has to be calculated.

Two FA and two HZ filter boxes are installed in parallel for redundancy purposes (see Figure 7). In a conservative approach, all the activity will be considered to be trapped in the filters located in the FA and HZ filter boxes closest from the wall of interest, i.e. no distribution between both redundant systems in parallel is taken into account.

For the wall sizing, the dose rate calculation is made at two calculation points as presented in Figure 7 . Both points are at $0.50 \mathrm{~m}$ of the wall and at $1.30 \mathrm{~m}$ high for the reasons previously exposed in Section 3.1. Point 1 is placed at the centre of the considered HZ filter box while Point 2 is at the centre of the FA filter box of interest.

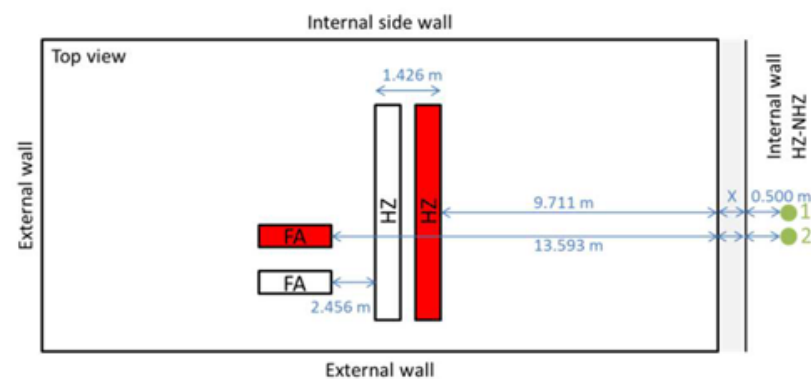

Figure 7. Schematic drawing of the calculation points in the EOF for internal wall sizing (top view). In red, the FA and HZ filter boxes used for dose rate calculations.

For the floor sizing, a similar approach is applied with Points 3 and 4 as shown in Figure 8. Point 3 is placed below the centre of the FA filter box while Point 4 is placed below the centre of the HZ filter box. Both are placed at $1.3 \mathrm{~m}$ high from the basement floor.

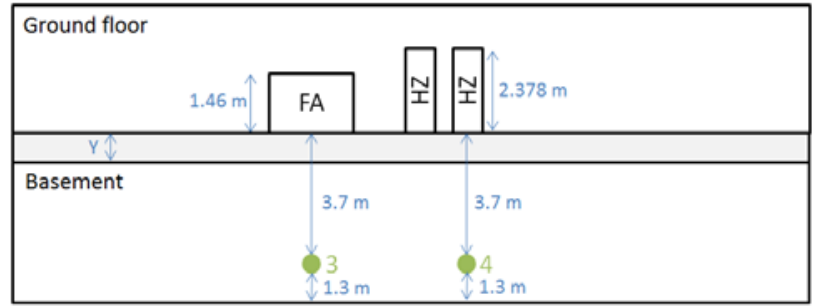

Figure 8. Schematic drawing of the calculation points in the EOF for floor shielding sizing (side view).
Note that these four calculation points are not related to those considered in Section 3.2. The shielding sizing will be established using the sum of the dose rates calculated at Points 1 and 2 for the wall and at Points 3 and 4 for the floor. Furthermore, for each of these calculation points, the dose rate results from the contribution of two source terms (a source term for absolute filters and a source term for charcoal filters).

\subsection{Materials}

The shielding is made of concrete (internal wall and floor) and of 304L stainless steel (external wall of the filter boxes). The material definition for the source terms is more complicated. For the absolute filters, it is a homogeneous mix of air (spaces between filters), 304L stainless steel (filter frame) and fiberglass paper, supposed to be $100 \%$ fiberglass for conservative purposes. For the charcoal filters, it is a homogeneous mix of air (spaces between filters), 304L stainless steel (filter frame) and carbon.

\subsection{Source terms}

The filters are supposed to be changed after 30 days of use following the occurrence of a severe accident. Note also that during the first venting, the ventilation system is kept closed to limit filter contamination. Consequently, to know the activity of the filters, the accumulation of particulates and iodine elements during this 30 day period needs to be determined. However, the temporal evolution of the volume activities around the EOF is established only for the first 10 days after severe accident (see Section 2). So, it was required to develop a methodology to calculate the accumulation beyond those 10 days. No radioactive decay of the filter accumulations is considered in order to be conservative and to simplify calculations. Furthermore, the release through natural leaks of the containment (second scenario in Section 2), which can not be neglected unlike for the external walls sizing, has a radically different behavior compared to the release from the filtered vent (first scenario in Section 2), meaning that two different approaches must be considered.

For the release through Natural Leak (NL), radionuclides with relatively long half-life show a volume activity around the $\operatorname{EOF}\left(A_{v, i}^{N L}(t)\right)$ which is approximately constant during the first ten days. Activity accumulation in the filters of the FA box is then calculated as follows [5]:

$$
A_{a c c, i}^{F A, N L}(\Delta t)=\frac{R_{\max }}{\lambda_{i}} \cdot\left(1-e^{-\lambda_{i} \cdot \Delta t}\right)
$$

With:

- $\mathrm{i}$, being representative of the studied radionuclide,

- $R_{\max }$, a constant representing the accumulation of radionuclides on the filters per second, arbitrarily and conservatively defined by the maximum of the activity around the EOF released through the NL during the studied period, multiplied by the air flow rate $\mathrm{D}$ of the ventilation system $\left(R_{\max }=\operatorname{Max}\left(A_{v, i}^{N L}(t)\right) . D\right)$,

- $\Delta t$, the time after the severe accident, 
- $\lambda_{i}$, the decay constant of the radionuclide $i$.

For the release through Filtered Vent (FV), radionuclides show a volume activity around the EOF $\left(A_{v, i}^{F V}(t)\right)$ which is very high during the first venting and rapidly drops during the next ventings. The formula, used to determine the activity accumulation of each radionuclide after a time $\Delta \mathrm{t}$ in the filters of the FA box, is the following one:

$$
A_{a c c, i}^{F A, F V}(\Delta t)=\left\{\begin{array}{c}
0 \text { if } \Delta t \leq t_{i} \\
\sum_{t=t_{i}}^{\Delta t} A_{v, i}^{F V}(t) . D .180 \text { if } t_{i}<\Delta t \leq t_{f} \\
A_{a c c, i}^{F A, F V}\left(t_{f}\right) \text { if } \Delta t>t_{f}
\end{array}\right.
$$

With:

- $\mathrm{i}$, being representative of the studied radionuclide, - $A_{v, i}^{F V}(t)$, the volumetric activity outside the EOF at a time $t$, released from the filtered vent,

- D, the air flow rate of the ventilation system,

- $\Delta t$, the time after the severe accident,

- $t_{i}$, the time when the ventilation system will open permanently after the first venting,

- $t_{f}$, the time after which the activity has reach a constant value and is considered as equal to $A_{a c c, i}^{F A, V}\left(t_{f}\right)$, this time being equal to 10 days.

The total activity accumulated on the FA filters is then the sum of the ones determined with formulas (1) and (2). Both previous formulas consider a maximum filtration efficiency of $100 \%$. Usually, filtration efficiencies used for the Tihange NPP site are 99\% for absolute filters and $94 \%$ for charcoal filters. Consequently, the activity in the filters of the HZ filter box, being after the FA filter box, will be not null but clearly inferior to activities accumulated in the FA filter box. Activity accumulation in the HZ filter box is calculated by applying the following formula:

$A_{a c c, i}^{H Z}(t)=\alpha \cdot A_{a c c, i}^{F A}(t)$

With $\alpha$ being equal to 0.01 for absolute filters and 0.06 for charcoal filters.

The list of radionuclides to be considered is decreased following the aforementioned methodology [3]. From the initial list of 1038 radionuclides, 11 radionuclides are considered for the dose rate calculations, six of them being iodine isotopes. Furthermore, considering the fact that the two types of filters in each box are trapping different radionuclide types, two source terms are to be considered: radioactive particulates for the absolute filters and iodine isotopes for the charcoal filters. Both of those filters are integrated in the FA and HZ filter boxes. As there are two source terms (iodine and particulates) to take into account for each calculation point (Points 1 and 2 for the wall and Points 3 and 4 for the floor) and as the MicroShield code does not enable the modeling of more than one source term per calculation point, eight dose rate calculations have to be performed for shielding thicknesses determination:

- At Point 1, dose rates due to the iodine isotopes in the charcoal filters and the radioactive particulates in the absolute filters of the HZ filter box,

- At Point 2, dose rates due to the iodine isotopes in the charcoal filters and the radioactive particulates in the absolute filters of the FA filter box,
- At Point 3, dose rates due to the iodine isotopes in the charcoal filters and the radioactive particulates in the absolute filters of the FA filter box,

- At Point 4 , dose rates due to the iodine isotopes in the charcoal filters and the radioactive particulates in the absolute filters of the HZ filter box.

As a consequence, eight specific models of calculations have to be developed and applied in order to determine, via dose rate calculations, the shielding thicknesses for the wall and the floor. These eight models are presented in the next section.

\subsection{Models}

A model includes the shielding (with all the elements between the source and the calculation point) and the source volume geometries. As previously mentioned, a total of eight models of calculation have to be established. Only a few of those models will be detailed here for illustration purposes.

Model 1 and Model 2 are used to calculate the dose rates at Point 2 due to iodine isotopes (as illustrated in Figure 9) and particulates in the FA filter box. Note that some volumes in Figure 9 are too thin to be identifiable on this drawing. Beside the source composition and density, the main difference between both models is the position of Point 2 compared to the source volume; the height of Point 2 being fixed and the height of the filters being different following the type (charcoal or absolute filters, see Figure 6). The source volume in Figure 9 (first volume on the left) has the dimensions of the charcoal filters for iodine isotopes but also of the absolute filters for the radioactive particulates, as found in the FA filter box.

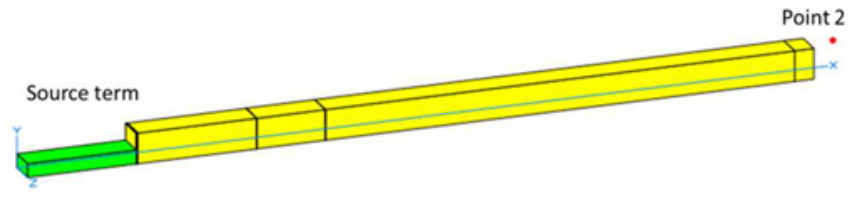

Figure 9. Representation of Model 1 used in MicroShield.

Several shielding layers are considered between this source volume and the Point 2, with some simplifications made in a conservative way:

- The shielding elements present between the source and the Point 2 are the wall of the FA filter box, the four walls of the two HZ filter boxes, the internal wall to be sized and the air between those elements,

- The HZ filter boxes are supposed to contain only air (which is a conservative assumption since air has a lower shielding effect than the materials which are really present),

- The two walls of a HZ filter box are combined into one because the number of shielding in MicroShield is limited ( $6 \mathrm{~mm}$ thick instead of two $3 \mathrm{~mm}$ thick walls).

Model 3 and Model 4 are used to calculate the dose rates at Point 1 due to iodine isotopes and radioactive particulates (as illustrated in Figure 10) in the HZ filter box. As for previous models, the main difference between Model 3 and Model 4 is the position of Point 1 compared to the source volume (first volume on the left in Figure 
10). For these models, the shielding elements present between the source and Point 1 are the wall of the HZ filter box, the internal wall to be sized and the air between those elements.

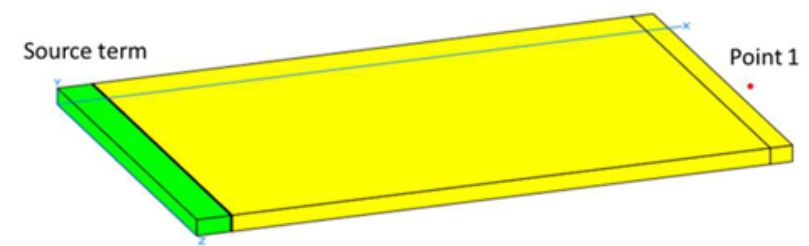

Figure 10. Representation of Model 4 used in MicroShield.

For the floor sizing, model definitions are similar to the ones for the wall. The source volume has the dimension of the FA or HZ filters. All shielding elements between the source and Point 3 or 4 must be considered. An example is shown in Figure 11, which presents the model used for calculation of the dose rate.

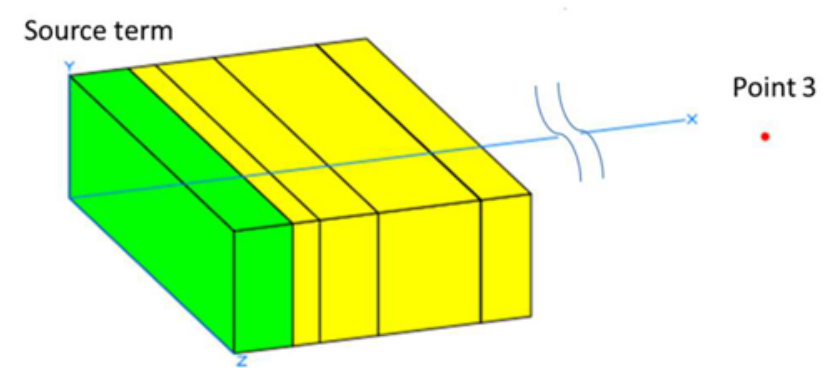

Figure 11. Drawing of the model used in MicroShield to define dose rate at Point 3 when the source term is the absolute filters of the FA filter box (the $\mathrm{x}$ axis being the vertical one).

In this example, the source volume (first volume on the left in Figure 11) has the dimensions of the absolute filters installed in the FA filter box. The different layers of shielding considered for this model are:

- The air between the absolute and charcoal filters inside the FA filter box (see Figure 6),

- The charcoal filters,

- The air between the charcoal filters and the bottom wall of the FA filter box,

- The steel for the bottom wall of the FA filter box (too small to be identified on the drawing),

- The concrete for the floor to be sized,

- The air down to Point 3 (localised at $1.3 \mathrm{~m}$ above the basement floor).

\subsection{Results}

For the internal wall sizing, calculations for the total dose rate provide a value of $0.0215 \mathrm{mSv} / \mathrm{h}$ without any shielding (i.e. wall thickness equal to $0 \mathrm{~cm}$ ). Consequently, this dose rate is below the required $0.26 \mathrm{mSv} / \mathrm{h}$ which makes the shielding useless at this location. No consideration on door thickness is needed since the wall will not ensure any shielding purposes.

For the concrete floor sizing, Figure 12 shows total dose rates calculated for several floor thicknesses. A curve is fitted on those calculated points and its intersection with the sizing criterion line $(0.26 \mathrm{mSv} / \mathrm{h})$ provides a minimum thickness of $12.7 \mathrm{~cm}$. However, the fitting function does not seem to be perfectly aligned with all the points. Therefore, to be conservative, a slightly higher concrete thickness of $13.5 \mathrm{~cm}$ might be more adequate.

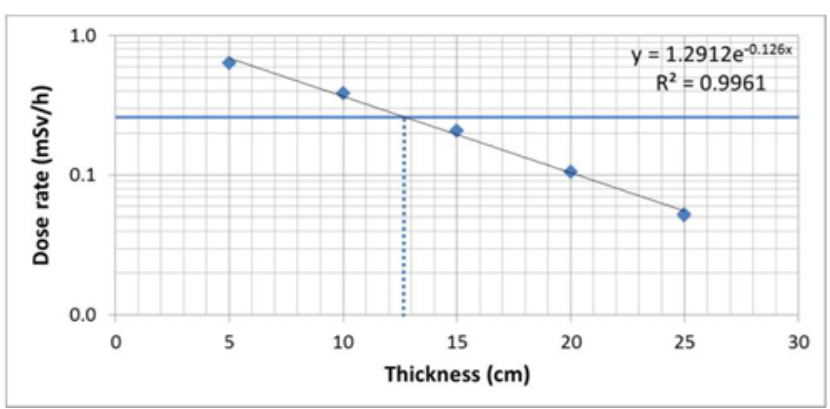

Figure 12. Total dose rate evolution relative to the floor concrete thickness (the horizontal line being the $0.26 \mathrm{mSv} / \mathrm{h}$ sizing criterion).

\section{Conclusion}

In this document, several methodologies are proposed for sizing the internal and external walls, floor, roof and doors of an Emergency Operation Facility (EOF) dedicated to manage a severe accident in a nuclear power plant. The particularity of such facilities is that the radioactive sources are expected both around and inside the building (considering the ventilation system).

All the proposed methodologies use the MicroShield code which is, usually, less adapted for complex geometries. The source term definitions and the models used for the shielding calculations with this code were developed and are illustrated, in this document, by calculations performed for the basic design of a new EOF for the Tihange nuclear power plant.

In that framework, it was considered that effective dose rate inside the habitable zone of the EOF had to be kept below $1 \mathrm{mSv} / \mathrm{h}$ after a severe accident, taking into account radioactive sources from inside and outside the building. Such a dose rate is achievable if a minimum thickness of about $50 \mathrm{~cm}$ for external walls and roof is considered. In addition, results have shown that internal shielding was not necessary, except for the floor where the HVAC filters are localised.

\section{References}

1. MELCOR Computer Code Manuals, Version 1.8.6, NUREG/CR-6119, Rev. 3, Sandia National Laboratories, Albuquerque, N.M. (2005).

2. Grove Engineering, MicroShield v5.05, Rockville, Maryland, USA (1999).

3. G. Genard, B. Gonze, D. Hulhoven, S. Vanderperre (Tractebel), How to correctly choose the list of relevant radionuclides to assess dose uptake by workers?, 2016 ISOE International Symposium (2016).

4. Grove Engineering, MicroShield v8.03, Rockville, Maryland, USA (2008).

5. Kenneth S. Krane, Introductory Nuclear Physics (John Willey \& Sons, Sommerset, New Jersey, 1987). 